\title{
$\mathrm{X}$.
}

\section{Bericht über Prof. Dr. Julius Böke's Ambulatorium für Ohrenkranke im Rochusspital im Jahre 1885.}

\author{
Von \\ Sigismund Szenes \\ in Budapest.
}

Im Jahre 1885 wurden an dem unter Leitung des Herrn Prof. B ök e stehenden Ambulatorium 868 Ohrenkranke (526 männliche, 342 weibliche) behandelt $=60,6$ Proc. männl., 39,4 Proc. weibl.

Interessanter sind die beziiglich des Alters gewonnenen Erfahrungen, aus denen hervorgeht, dass mit zunehmendem Alter auch die Zahl der Kranken wächst, jedoch nur bis zum 30. Jahre; von da $\mathrm{ab}$ sinkt sie wieder. Die meisten Kranken (284) waren 20 bis 30 Jahre alt. Die Zahl der Erkrankungen ist eine grössere als die der Patienten, da ein und derselbe Patient oft $2-3$ von einander vollkommen unabhängige Erkrankungen darbot.

Erkrankungen des äusseren Ohres kamen 233 mal vor (141 M., 92 W.). - Abscessus auriculae sin. 1 (M.), Cysta auric. sin. 1 (M.), Fistula auric. dextr. 1 (M.), Perichondritis auric. dextr. 1 (W.), Erysipelas auric. dextr. 1 (M.), Bildungsanomalie der Muschel 1 (M.), Eezema 20 (6 M., 14 W.), Furunculus in meatu auditorio externo 47 (25 M., 22 W.), Cerumen obturans 143 (93 M., 50 W.), Corpus alienum in meatn auditorio externo 11 (8 M., $3 \mathrm{~W}$.), Otitis externa 6 (4 M., 2 W.).

Als Bildungsanomalie der Muschel möge der folgende Fall mitgetheilt werden. Ein 12 jähriger Schüler sprach mit der Klage vor, dass sein rechtes Ohr seit mehreren Monaten zeitweilig sause und das Gehör an diesem $\mathrm{Ohr}$ auch schwächer sei. Die Untersuchung constatirte chronischen Katarrh der Trommelhöhle, der sich auf Behandlung besserte. Bei diesem Kranken, der sonst ziemlich gut entwickelt war und dessen 3 Geschwister gesund sind, war linkerseits blos ein rudimentäres Ohrläppchen zugegen; die Oeffnung des äusseren Gehörgangs fehlte. Linkerseits war sowohl der Ober-als Unterkiefer in seiner Entwicklung zurückgeblieben, der untere linke Augenzahn stand hinter dem Incisivus, das linke Auge stand höher als das rechte, die linke Nasenöffnung war kleiner als die rechte, desgleichen die linke Pupille kleiner, was namentlich bei der Accommodation auffält. Die von Prof. Laufen a uer vorgenommenen Schädelmessungen erwiesen sich als normale, und war blos eine Asymmetrie des Gesichtes zugegen, die anf Atrophie der linken Gesichtshälfte zurückzuführen war. Diese Asymmetrie war anch am Rumpf und an dem rachitischen Thorax sichtbar. Nebst diesen Veränderungen fehlte das Gehör linkerseits.

Bezüglich des $\mathrm{Ekzems}$ ist zu bemerken, dass es bei Frauen 
hänfiger war, offenbar deshalb, weil das $\mathrm{Ohr}$ beim weiblichen Geschlecht, durch das lange Haar, häufiger Irritationen ansgesetzt ist als beim männlichen. Als gutes Mittel erwies sich, selbst in den hartnäckigsten Fällen, das $01 \mathrm{eum}$ cadini.

Beim Furnnkel fiel es auf, dass er beiderseitig zugleich blos in 3 Fallen, links hingegen bedeutend häufiger als rechts zu finden war. Die Therapie bestand in tiefer Incision. Wo zu dieser die Kranken nicht einwilligten, in warmen Kataplasmen.

Cerumen obturans wurde in dem linken Gehörgang öfters gefunden als rechts und beiderseitig, jedoch ist der Unterschied nur ein geringer. Beziuglich des Alters wächst die Zahl der Erkrankungen zwar bis zum 30. Jahre, um dann zu sinken; im 40.-50. Jahre ist jedoch wieder ein Wachsen zu bemerken. Die Therapie war: Ausspritzen des Ohres; gelang dies nicht, so wurde die Beförderung des Ohrenschmalzpfropfes nicht forcirt und der Kranke bekam eine 1 proc. Natr. bicarb.-Lösung $(0,4 / 40,0)$ mit welcher er das $\mathrm{Ohr} 2-3$ stiindlich lauwarm füllte, um die Masse zu erweichen, welche bei dem nächsten Kommen dann ausgespritzt wurde.

Mit Fremdk ör rpern präsentirten sich 11 ( 8 M., 3 W.). Manche wurden von den Patienten behufs Heilzwecks ins Ohr gesteckt, andere gelangten während des $\mathrm{Spieles}$ dahin, wieder andere kamen zufällig ins Ohr. Im Allgemeinen bestand die Therapie in Ausspritzung des Ohres, wobei das ausfliessende Wasser den fremden Körper gewöhnlich mit sich riss. Als fremde Körper figurirten: Knoblauch, Kukurutz, Stroh, Zündhölzchenstücke etc. Zur Extraction griff man nur in den seltensten Fallen, dann namentlich, wenn der Fremdkörper unangenehme Sensationen verursachte. So lange der Fremdkörper nicht gesehen wird, möge der Gehörgang ein noli me tangere bleiben, und ist namentlich bei Extractionen die grösste Vorsicht zu gebrauchen. Oft verursachten Mittelohraffectionen solche Empfindungen, als wäre ein Fremdkörper im $\mathrm{Ohr}$, und selbst in einem derartigen Fall kann der praktische Arzt durch ein vorsichtiges Ausspritzen nicht so viel sehaden als mit einer aufs Geradewohl effectuirten Extraction, bei welcher das $\mathrm{Ohr}$ gewöhnlich nur lädirt wird.

Bei der Otitis externa prävalirte die linke Seite. Die Therapie bestand in Warmbalten und Ruhe des Ohres, welche in den meisten Fällen von Erfolg war.

Die Erkrankungen des Mittelohres. Das grösste Contingent lieferten die Erkrankungen der in das Gebiet des Mittelohres gehörigen Organe, und beruht dies vornehmlich darauf, dass die scheinbar von der Aussenwelt abgeschlossene Trommelhöhle vermittelst Mund und Nase mit derselben in Contact steht und durch deren Vermittelung erkrankt, eine Heilung jedoch nur auf directe Behandlung einzutreten pflegt. So kann sich gelegentlich eines heftigeren Schnupfens ein Katarrh der Trommelhöhle entwickeln, der jedoch mit dem Schwinden des Schnupfens nicht sistirt und nur dann heilt, wenn er einer directen localen Behandlung unterzogen wird.

Es erkrankten an Myringitis 11 (10 M., 1 W.), Haemorrhagia inter substantiam membranae tympani 1 (M.), Abscessus membranae 
tymp. 5 (4 M., 1 W.), Ruptura membranae tymp. 8 (7 M., 1 W.), Cicatrices membr. tymp. 27 (14 M., 13 W.), Calcifiatio in membr. tymp. 3 (2 M., 1 W.), Catarrhus cavi tympani acutus $114(70$ M., 44 W.), Cat. chron. cav. tympani 258 (152 M., 106 W.), Otitis media acuta 48 (33 M., 15 W.), Otitis media chronica purulenta 189 (113 M., 76 W.), Periostitis process. mastoidei 7 (6 M., 1 W.), Polypus 13 (8 M., 5 W.), Otalgia tympanica 16 (6 M., 10 W.).

Eine selbständige Erkrankung des Trommelfells kommt selten vor, insgesammt gelangten 11 Fälle von Myringitis zur Beobachtung ( 10 M., 1 W.), da solehe ambulatorische Kranke, weil der ärmeren Klasse angehörig, nicht allsogleich ärztliche Hülfe aufsuchen, sondern erst dann vorsprechen, wenn die üblichen Hausmittel versagten und es bereits zn Eiternng kam; für die häufigere Erkrankung des männlichen Geschlechtes spricht der Umstand, dass es äusseren Noxen hänfiger ausgesetzt ist als das weibliche. Die Behandlung war eine antiphlogistische. Blutegel anf den Processus mastoideus, ein Antipyreticum, und behufs Stillung der Schmerzen ein Narcoticum in Form einer Salbe, einzureiben auf die das kranke $\mathrm{Ohr}$ umgebenden Hautstellen (Extr. opii aqu. 0,4, Ung. hydrarg. cin. 10,0). Eine solche Behandlung geniigte. Kommt es zur Abscedirung, dann sind warme Kataplasmen, Punction und nachherige Ausspülung mit einer lauen antiseptischen Fliissigkeit am Platze.

Rupturen des Trommelfells infolge Tranma kamen 8 mal ( $7 \mathrm{M}$., 1 W.) vor. In einem Falle handelte es sich um einen 32 jährigen Kutscher, der vor 8 Tagen vom Pferde auf das rechte 0 hr gefallen war, worauf er auf kurze Zeit bewusstlos ward; beim Erwachen bemerkte er einige aus dem Ohr fliessende Bluttropfen. An diesem Ohr ist das Gehör geschwächt und Sausen zugegen. Bei der Untersuchung fand sich im oberen Viertel die Verletzung, die den Eindruck machte, wie wenn das Trommelfell an dieser Stelle von seiner Peripherie abgerissen wäre; am Griff des Hammers waren die Gefässe ein wenig injicirt, der Lichtkegel jedoch war vorhanden. Anf Wärme, Ruhe und Opiatsalbe vernarbte die Stelle bald. - Ein anderes Mal stellte sich eine 43 jährige Frau vor, die vor 3 Wochen von ihrem Mann eine Ohrfeige erhalten hatte und seither schwer hört, Schmerzen empfindet und seit 2 Wochen einen eitrigen Ausfluss beobachtet. In der oberen Hälfte des linken Trommelfells sind zwei kreisförmige, mit Eiter gefuillte perforirte Stellen sichtbar. Auf Zinkessigsolution $(0,4: 40,0)$ sistirte der Ausfluss bald und nach 4 Wochen, als sich Patientin mit einem am Ohr sitzenden Furunkel vorstellte, waren beide Perforationen vernarbt. - Bei Rupturen geniigte oft ein hermetischer Verschluss der Ohröffnung; auf die Verletzung direct einwirkende Mittel wurden gemieden, weil sie die Heilung nur verzögern.

In einem Fall war Ruptur des Trommelfells mit Schädelbruch zugegen. Ein Arbeiter fiel mit seinem Kopfe auf einen prominenten Theil eines Dampfkessels auf, verlor das Bewusstsein und zeigte profuse Blutung ans dem rechten Ohr. Nach 14 tägiger Behandlung war die erlittene Kopfwunde noch nicht vernarbt, vielmehr sickerte ans derselben Secret hervor und war schmerzhaft. Bei seiner Auf- 
nahme auf die zweite chirurgische Abtheilung des Rochusspitales war an jener Stelle, wo sich das Seitenbein mit dem Schläfenbein vereinigt, eine nahezu hirsekorngrosse Oeffnung anzutreffen, aus der eine gelbliche Flïssigkeit hervorsickerte. Die Sonde drang $2 \mathrm{Cm}$. tief ein und der Knochen war rauh, uneben, ein wenig beweglich, es war demnach ein Knochenbruch zugegen. Die Untersuchung constatirte ferner eine 'gleichzeitige Facialislähmung peripheren Ursprungs. Patient sprach nach einer glïcklich überstandenen Trepanation auf dem Ambulatorium vor, wo man das rechte Trommelfell nach aussen hervorgewölbt, in seinem oberen Theil durch eine streifenförmige, weisse Narbe verflacht, blänlich verfärbt fand, woraus man auf die Gegenwart eines im Cavum tympani vorhandenen und das Trommelfell zum Theil deckenden Blutschollens schliessen konnte. Die in die Eustach'sche Röhre eingeblasene Luft erzeugte keine Schwingungen, woraus wieder hervorging, dass der Blutschollen weder von Flüssigkeit noch von flüssigem Blut umgeben war. Patient hörte das Ticken der $\mathrm{Uhr}$ auf $5 \mathrm{Cm}$, das leise gesprochene Wort auf 3 Meter Distanz; der Hörnerv war demnach intact und der bestehende stumpfe Gehörsinn war. blos durch das Ergriffensein eines Theiles des schalleitenden Apparates bedingt. Bezüglich der Facialislähmung war ans der Intactheit des Acusticus nahezu bestimmt der Schluss zu ziehen, dass der Facialis an einer solehen Stelle lädirt war, wo er den Acusticus schon verliess, dass demnach eine Ruptur oder Verletzung weder im Apex des Labyrinths noch an dessen Grunde stattfand, hingegen konnte eine solche an dem Tegmen tympani oder in der Gegend der Membrana tymp. stattgefunden haben, und dass das ansgetretene Blut, infolge eines Druckes auf den Nerv. facialis, dessen Function behinderte.

Bei einem 23 jährigen Tischler gesellte sich zur Verletzung des Trommelfells Functionsstörung des Labyrinths. Der Betreffende fiel vor 11 Tagen von 4 Meter Höhe herab und blieb bewusstlos liegen; als er zu sich kam, bemerkte er, dass er mit dem linken Ohr schwächer hörte und dass aus demselben Blut sickerte. Bei der Untersuchung fand sich im hinteren oberen Quadranten des Trommelfells nebst lebhafter Röthe eine nahezu 2,5 Mm. lange Ruptur vor. Auf Ruhe und die bereits angeführte Salbe liess die Injection am 5. Tage nach. Tags darauf verliess Patient das Spital, und wiewohl die Verletzung noch nicht verheilt war, begann er zu arbeiten. Nach 3 Wochen sprach er wieder vor; dazumal war die Perforation beträchtlich verkleinert, doch der Schwindel stellte sich zeitweilig noch ein. Das Gehör war so geschwächt, dass Patient das Ticken đer Uhr nur beim Andrïcken an die Muschel zu hören vermochte; die Untersuchung mit der Stimmgabel erschloss ferner, dass infolge des Falles auch eine Störung im Labyrinth auftrat; auf die Stirne gelegt, vermochte Patient das Tönen der Stimmgabel nur mit dem intacten $\mathrm{Obr}$ zu hören. Nebst Ruhe wurde Patient innerlich eine 5 proe. Bromsolution ordinirt.

Na rb en am Trommelfell kamen in 27 Fällen vor (14 M., $13 \mathrm{~W}$.); kalkige Ablagerungen 3 mal. 
Acuter Katarrh der Trommelhöhle kam 114 mal vor $(70 \mathrm{M} ., 44 \mathrm{~W}$.$) , zumeist beiderseitig. Am häufigsten wurde diese$ Erkrankung im Alter von 20-30 Jahren beobachtet (27 M., $16 \mathrm{~W}$.). Nahezu in allen Fällen klagten die Betreffenden über Sausen, undeutliches Gehör und zeitweiliges Verstopftsein des Ohres. Auf Anwendung des Politzer'schen Verfahrens sehwanden diese subjectiven Erscheinungen, oft wie auf einen Zauberschlag. In einem Fall war der Katarrh mit Facialislähmung complicirt. Beide Erkrankungen heilten nach kaum einwöchentlicher Behandlung nach Lufteintreibungen nach dem Politzer'schen Verfahren.

Chronischen Katarrh der Trommelhöhle beobachtete man $258 \mathrm{mal}$ ( $152 \mathrm{M}$., $106 \mathrm{~W}$.), ebenfalls zumeist beiderseitig (162); die häufigsten Erkrankungen (76) kamen im Alter von 20-30 Jahren vor. - $7 \mathrm{mal}$ war circumscripte Atrophie des Trommelfells zugegen. Ein 70 jähriger Maschinist klagte über stets zunehmende Schwäche seines Gehörs. Bei der Untersuchung zeigten sich beide Trommelfelle verdickt, vom Lichtkegel keine Spur; rechterseits entlang des Hammergriffes injicirte Gefässe; linkerseits eine den ganzen oberen hinteren Quadranten in sich fassende Atrophie. - Dieser katarrhalişche Zustand der Trommelhöhle blieb anch auf den Musc. tensor tympani nicht ohne Einwirkung, was sich in einer Einknickung des Trommelfells manifestirte. Ein anderes Mal hinwieder wurde der Hammer so weit nach aussen gehoben, dass das Trommelfell den Eindruck machte, wie wenn es sich ektropionirt hätte und durch den Griff des Hammers in zwei Theile getheilt wäre. In 2 Fällen war Spasmus dieses Muskels zugegen; bald verschwand der Lichtkegel, bald wieder $\mathrm{kam}$ er zum Vorschein. In einem Fall gesellte sich dem chronischen Katarrh Facialislähmung bei. Je nach dem Fall kam der Politzer'sche Ballon oder der Katheter zur Anwendung; ansserdem wurde behufs Resorption des im Cavum tympani aufgehäuften Serums Jodsalbe ordinirt, bei katarrhalischer Affection der naso-pharyngealen Schleimhaut Gargarismen.

Acute Entzündung der Trommelhöhle kam 48 mal vor (33 M., 15 W.). Eigenthïmlicherweise war diese Erkrankung beiderseitig nicht zu beobachten; rechts war sie hänfiger als links. Auch hier participirte zumeist das Alter vom 20.-30. Lebensjahre. Therapie: Blutegel, Ruhe, Warmhalten des Ohres, Ung. ciner. c. narcotico.

Viel häufiger beobachtete man die chronische Entzündung der Trommelhöhle, $189 \mathrm{mal}$ (113 M., $76 \mathrm{~W}$.); linkerseits und beiderseits nahezu in gleicher Zahl. Bis zum 30. Jahre war stete Zunahme dieser Erkrankung zu constatiren, von da ab rapides Sinken der Krankenzahl. Bei den Frauen entfiel die höchste Zahl (27) auf die $\mathrm{Pubertätszeit} \mathrm{und} \mathrm{scheint} \mathrm{jenes} \mathrm{Moment,} \mathrm{wonach} \mathrm{in} \mathrm{diesem} \mathrm{Zeit-}$ alter die Disposition zu Entzündungen eine grössere sei, auch für die Trommelhöhle Geltung zu haben. - Die Therapie bestand darin, dass die Trommelhöhle einer Wundfläche gleich rein gehalten, ausgespritzt und später mit Adstringentien behandelt wurde; ausserdem presste man behufs Vermeidung von Synechien Luft in die Trommelhöhle ein. Wird keine entsprechende Behandlung eingeleitet, dann 
kommt es zu mannigfaltigen Complieationen, so zu Facialislähmung, Excrescenzen, Atresien. - In 2 Fällen war eine croupöse Entzlindung der Trommelhöhle zn beobachten, welche auf warme Kataplasmen, später Ausspritzen mit 5 proc. Acidnm boricum-Solution und hernach auf Acet. zinci $(0,4: 40,0)$ heilte.

Als ernste Complication der eitrigen Entzündung der Trommelhöhle ist die Periostitis des Processus mastoideus zu betrachten. Solehe Fälle kamen 7 mal vor $(6 \mathrm{M}, 1 \mathrm{~W}$.) und erheischen ein fachgemässes, energisches Vorgehen, da im widrigen Fall eine cerebrale Affection sich bald hinzugesellt. Eisbentel, ein Laxativum, innerlich Chinin, später Priessnitzumschläge, Einpinselung mit Tinct. jodi führten stets zur Heilung.

Eine fernere Complication bildete noch der Polyp, der $13 \mathrm{mal}$ (8 M., 5 W.) zur Beobachtung gelangte. Behufs Heilung des Grundubels ist stets die Entfernung, resp. Verkleinerung des Neoplasma anzustreben. Ersteres geschah stets mit dem Wilde'schen Polypenschniirer, die Stelle wurde dann mit Arg. nitr. geätzt; Verkleinerung wurde bei Anwendung von Alcohol. abs., gänzliches Schwinden bei Anwendung von Acid. chromicum erreicht.

Als in den Rahmen der Erkrankungen des Mittelohres gehörend sind noch anzuführen die 0 talgia tympanica, die von den im Carum tymp. verlaufenden Nerven ausgeht, so unter anderen ein Reizzustand der hier verlanfenden Trigeminuszweige. Bei Intactheit des Gehöres charakterisiren sich diese Erkrankungen blos durch den intensiven Schmerz. Oft löst ein cariöser Zahn diese Schmerzen aus; die anf Extraction des Zahnes aufhören. Wo derartige Ursachen nicht nachweisbar waren, griffen wir behufs Sistirung der Schmerzen zu Chinin, Natrium salicyl., Bromkali. Diese nervösen Zustände kamen $16 \mathrm{mal}(6 \mathrm{M}$., $10 \mathrm{~W}$.) vor und sassen sie nicht immer im Mittelohr allein, sondern griffen anch anf andere Körpertheile ïber. In solchen Fällen bewährte sich Bromnatrium.

Erkrankungen des inneren Ohres. Insgesammt 28 Falle (18 M., 10 W.). Diese geringe Zahl erklärt sich dadurch, dass das innere $\mathrm{Ohr}$ vor Noxen besser geschitzt ist, als das mittlere oder äussere; auf dieser Unzugänglichkeit beruht auch der geringere Erfolg der Therapie. In obiger Zahl participiren die Tauben mit 7 (4 M., 3 W.), die Taubstummen mit 12 ( 7 M., 5 W.). Bei den ersteren waren die veranlassenden Ursachen Meningitis, Typhus; bei den letzteren war die Erkrankung $6 \mathrm{mal}$ angeboren, 2 mal durch Typhus, 4 mal durch Meningitis, resp. Eklampsie bedingt. 3 mal wurde bei Männern Labyrinthitis diagnosticirt, je 1 mal eine Hyperacusis nervosa und eine Irritatio labyrinthi; Hyperämie des Labyrinths, ebenso eine Commotion desselben wurde in je 2 Fällen ( 1 M., 1 W.) gefunden.

Insgesammt wurden daher bei den 868 Ohrenkranken (585 M.,

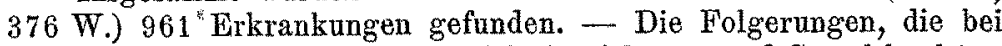
den einzelnen Krankheiten bezüglich des AIters und Geschlechtes gemacht wurden, sollen im weiteren Beobachten ibre Bekräftigung finden. 